mortality risk. The causes of death included dissection of the aorta and pneumonia (nonepilepsy related), and accidental drowning, and SUDEP. Five of the patients with clusters during treatment died, whereas none with pre-treatment clusters died. (Sillanpaa M, Schmidt D. Seizure clustering during drug treatment affects seizure outcome and mortality of childhood-onset epilepsy. Brain April 2008;131:938-944). (Respond: Prof Dr Dieter Schmidt, Epilepsy Research Group, Goethestr 5, D-14163 Berlin, Germany, E-mail: dbschmidt(at-online.de).

COMMENT. Clustering of seizures during treatment is associated with a less favorable long-term outcome compared to clustering prior to treatment. Patients with seizure clustering during treatment compared to those without are four times more likely to have drug resistant epilepsy and an increased risk of mortality. Patients with clustering before beginning treatment is not associated with a poor prognosis. Clustering was not associated with status epilepticus in this study. The authors favor trials of aggressive treatment of seizure clustering.

\title{
SCREENING TEST FOR DRAVET SYNDROME BEFORE ONE YEAR
}

Risk factors for Dravet syndrome were determined in 96 children who experienced febrile seizures before age one year, in a retrospective study at Okayama University and other centers in Japan. Clinical characteristics were compared in 46 patients who had developed Dravet syndrome and 50 without the syndrome. Significant risk factors included an age of onset of febrile seizure $<7$ months, a total of $>5$ seizures, and prolonged seizures $>10 \mathrm{~min}$. Other highly predictive factors were hemiconvulsions, partial seizures, myoclonic seizures, and hot water-induced seizures. A total clinical score of 6 or above was the cut-off value for a high risk of Dravet syndrome. (Each risk factor was assigned a score of 0-3, based on the p-value; $>5$ seizures [3], hemiconvulsion [3], prolonged seizure [3], onset $<7$ mos [2], hot water-induced seizure [2], focal or myoclonic seizure [1]). SCN1A mutations were detected significantly more often in the Dravet group (41-43\%) than in the non-Dravet syndrome group (0-12\%) of patients. (Hattori J, Ouchida $\mathrm{M}$, Ono $\mathrm{J}$ et al. A screening test for the prediction of Dravet syndrome before one year of age. Epilepsia 2008;49(4):626-633). (Respond: Dr Iori Ohmori, Department of Cellular Physiology, Graduate School of Medicine, Okayama University, 5-1 Shikata-cho, 2-chome, Okayama 700-8558, Japan. Email: iori(a.md.okayama-u.ac.jp).

COMMENT. In this practical screening test for the differentiation of Dravet syndrome from febrile seizures, if the patient has a clinical risk score of 6 or more, there is a high risk of Dravet syndrome. SCN1A mutation analysis is recommended if available in infants with a risk score of $-1>6$. Dravet syndrome or SMEI (severe myoclonic epilepsy of infancy), an intractable form of epilepsy, is difficult to differentiate from a febrile seizure disorder before the first birthday. Seizures are febrile hemiclonic or generalized tonic-clonic, frequently recurrent and prolonged, and are complicated by status epilepticus during infancy. Myoclonic, focal, absence and atonic seizures evolve between 1 and 4 years, and are accompanied by slow development and regression. Neurologic abnormalities include spasticity, ataxia and cognitive impairment. SMEI is one of a spectrum of infantile epileptic encephalopathies with SCN1A mutations. (see Ped Neur Briefs April 2007;21:25-26). Early 
diagnosis should allow more accurate parental counseling and more effective long-term treatment.

\section{ABORTED AND REFRACTORY STATUS EPILEPTICUS COMPARED}

Clinical and EEG characteristics, etiologies, treatment response, and predictors of long-term outcome were determined in 154 children with status epilepticus (SE) hospitalized at the Mayo Clinic, Rochester, MN, 1994-2004. Patients with status aborted with medication (ASE) in $69 \%$ were compared to $39 \%$ with refractory SE (RSE). SE was defined as continuous tonic-clonic or electrographic seizure activity for at least $10 \mathrm{~min}$ or intermittent seizure activity without recovery of consciousness for at least $30 \mathrm{~min}$. (Mayer SA et al. Arch Neurol 2002;59:205-210). Etiology of SE was acute symptomatic in 26\%, remote symptomatic $35 \%$, idiopathic $20 \%$, and febrile in $10 \%$. RSE compared to ASE was significantly associated with a higher family history of seizures, higher number of seizures and AEDs, nonconvulsive SE, and focal or electrographic seizures on initial EEG. In-hospital mortality was significantly higher with RSE $(13.3 \%)$ than ASE $(2.1 \%)$. RSE patients developed more neurological deficits and more epilepsy at long-term follow-up than ASE children. More aggressive treatment resulted in better responses and outcomes. Poor outcome risk factors included long seizure duration, acute symptomatic etiology, nonconvulsive SE, and young age ( $<5$ years) at admission. Prospective, randomized trials of different treatment protocols are advocated. (Lambrechtsen FACP, Buchhalter JR. Aborted and refractory status epilepticus in children: a comparative analysis. Epilepsia 2008;49(4):615-625). (Respond: Jeffrey R Buchhalter MD, Phoenix Children's Hospital, 1919 E Thomas Road, Phoenix, AZ 85016).

COMMENT. Status epilepticus in children is refractory in $40 \%$, and RSE is related to family history, number of seizures and AEDs, nonconvulsive status, and initial EEG abnormalities. Etiology is an important determinant of outcome, especially acute symptomatic causes. Identification of these risk factors should lead to more aggressive therapy and better outcome.

\section{INFECTIOUS DISORDERS}

\section{HERPES SIMPLEX VIRUS-1 AND BELL'S PALSY}

The association between herpes simplex virus-1 (HSV-1) infection and Bell palsy was determined in 47 children studied at Children's Hospital at Montefiore, Bronx, NY. Swabs of saliva and conjunctiva were taken for PCR testing. To validate PCR testing, swabs were obtained from patients with oral lesions of herpes gingivostomatitis. An HSV-1 enzyme-linked immunosorbent assay was positive in 33 of 42 affected patients compared to 16 of 41 controls $(\mathrm{P}=0.0003)$. HSV-1 polymerase chain reaction was positive in 10 of 47 affected patients compared to 4 of 45 controls $(\mathrm{P}=0.08)$. The findings support an association between HSV-1 infection and Bell palsy in children. (Khine H, Mayers M, Avner JR, Fox A, Herold B, Goldman DL. Association between herpes simplex virus-1 infection and idiopathic unilateral facial paralysis in children and adolescents. Pediatr Infect Dis J May 